\title{
Data Mining
}

\section{This paper, selected for the category "Best papers from 1959 to 2008", was first published in WIRTSCHAFTSINFORMATIK 35(5)1993:481-487.}

\section{DOI 10.1007/s12599-008-0005-4}

\section{The Authors \\ Dr. Nicolas Bissantz \\ General Manager \\ Bissantz \& Company GmbH \\ Nordring 98 \\ 90409 Nürnberg \\ Germany \\ nicolas.bissantz@bissantz.de \\ Dr. Jürgen Hagedorn \\ SAP AG \\ Hasso-Plattner-Ring 7 \\ 69190 Walldorf \\ Germany}

This article is also available in German in print and via http://www.wirtschafts informatik.de: Bissantz N, Hagedorn J (2008) Data Mining (Datenmustererkennung). WIRTSCHAFTSINFORMATIK. doi: 10.1007/11576-008-0108-z.

"The fruits of knowledge growing on the tree of data are not easy to pick." (Frawley et al. 1991)

\section{Terminology}

The term "data mining" describes the extraction of implicitly available, non-trivial and useful knowledge from large, dynamic databases with relatively complex structures. In this connotation, knowledge is defined as a pattern that 1.) is interesting in the eyes of the user and 2.) actually exists with adequate certainty. Patterns can describe relationships among data sets, relationships among the data in a single data record, or specific frequencies. The purpose of data mining, therefore, is to:

Some literature also uses terms such as 'knowledge discovery','database mining' or 'database exploration' to discuss the topic of data mining.
- identify these types of patterns,

- use them to derive rules,

- find groups among data sets,

- give these groups descriptive attributes,

- find representative examples,

- construct functions that apply for numerical variables.

Fig. 1 shows a sample rule that could be used in conjunction with a profit and loss statement.

The various methods in the field of data mining are generally characterized by the following traits:

- Users will receive the required information in an easy-to-understand form (e. g. a text or a chart). Alternatively, a program will further process this information.

- Measures are given to evaluate the reliability and certainty of the extracted knowledge.

- The generated information is not trivial compared to the knowledge that users or the system already have.

- The run time of the underlying algorithm is acceptable. Depending on the number of data sets being searched, this response time should not exceed that of a low-level polynomial.

The significance of data mining has steadily increased since the 1990's. As the number and size of databases continue to boom, however, users are discovering that analyzing these immense volumes of data is a highly complex task. Since this often involves "mixed" data sets that contain nominal and metric attributes, even statistical approaches are only suitable to a certain degree. As a result, the gap between generating and understanding data volumes continues to grow.

Data mining has since evolved into an independent field of research in which intelligent data analysis methods attempt to "unearth the buried treasures from the mountains of raw data". Users expect to gain a competitive advantage from this form of automated data preparation and analysis.

\section{Usage scenarios}

Data mining is widely used in a range of scientific disciplines and business scenarios. Some noteworthy examples include findings in the areas of database management, machine learning, Bayesian inference, knowledge gain for expert systems, fuzzy logic, neural networks, and genetic algorithms.

Examples in everyday business scenarios include database marketing for airlines, panel data research as well as the creation of customized trade publications based on subscriber data for hundreds of different user groups. Frawley and Piatetsky-Shapiro (Frawley et al. 1991) offer a detailed overview of further areas of usage.

Gross margin analysis is another interesting field of research in data mining. With the help of modern cost accounting software, companies can perform multidimensional analysis on individual income items. Fig. 2 lists a few sample questions related to this topic. Due to the numerous reference objects (e. g. products, customers, sales channels, regions) and the resulting number of objects that need to be examined, controllers require methods that automatically identify data patterns. In this case, these patterns are a combination of attribute values (e. g. "DIY stores" and "power drills" in Fig. 1) as well as measures (e. g. gross margin). A company that develops a data mining program must also consider the large volumes of data involved. Even in a midsize company, for example, it is common that several hundred-thousand items flow into a monthly income statement.

Case Based Reasoning (CBR) is one interesting example of how data mining and machine learning could work together. CBR components attempt to trace current questions to problems that have already been solved in the past. Help desks, which assist in clarifying the questions a customer has about purchased products, are one practical usage of this type of procedure. While some compa- 
nies use help desks to support their telephone hotlines, others give their customers direct access through a remote data transfer. Data mining can be very valuable in this context because it consolidates the information gathered in thousands of individual historical cases into key findings. The advantage of this procedure is the shorter process of searching for precedents which can be used to answer the current customer's question.

\section{Methods}

There are many different types of methods to analyze and classify data. Some common methods include cluster analysis, Bayesian inference as well as inductive learning. Cluster analysis can be used based on numerical measures as well as in the form of conceptual clustering.

The structures of data mining systems are very different by nature. The following configuration, however, is very common:

- The analysis method, which identifies and analyzes patterns, forms the core of the system.

- The input can include components such as raw data, information from a data dictionary, knowledge of the usage scenario, or user entries to narrow the search process.

- The output encompasses the found measures, rules or information which are presented to the user in an appropriate form, incorporated into the system as new knowledge or integrated into an expert system.

Figure 3 shows an overview of the concept described above ${ }^{2}$.

\subsection{Cluster analysis}

Whether in its traditional form or as conceptual clustering, cluster analysis attempts to divide or combine a set number of objects into groups based on the proximity that exists among these objects. The clusters are grouped so that there are large similarities among the objects of a class as well as large dissimilarities among the objects of different classes.

\subsubsection{Traditional cluster analysis}

Regardless of the scaling level of the object variables, there are multiple ways

\footnotetext{
Modified figure from Frawley et al. (1991).
}

If customer group $=$ DIY store and artcle $=\mathrm{HX7}$ power drill

then gross margin low $=$ true with probability $=.9$

Fig. 1 Sample rule in gross margin analysis

- Do shrinking gross margins and growing sales show that some sales reps change their discount policy?

-Which causes add to sales deviations and how strong are they? (price, volume, mix effects)

-Which combinations of products and customers produce negative gross margin?

Fig. 2 Sample issues in the field of gross margin analysis

to measure the similarity and difference of the proximity. Basic examples include the Euclidean (i. e. the square root of the total squared difference) and Manhattan differences (i. e. the sum of the absolute differences of individual variables). In general, we can examine metric, nominal as well as mixed data sets by varying the proximity measure.

When objects have different types of attributes, for example, Kaufman and Rousseeuw recommend calculating a difference of 0 for the individual nominal attributes when the values are the same, and a difference of 1 when they are different. In the case of metric variables, we first need to establish the difference among the object values. To standardize them we then divide them by the maximum difference. The result is a difference between 0 and 1. We then calculate the total difference between two object vectors as the sum of the individual differences (Kaufman and Rousseeuw 1990).

We can use this type of measure (eventually extended by the weight of an individual attribute) to cluster data sets in gross margin analysis. These contain nominal attributes (e. g. product, customer, region) as well as numerical measures (revenues or gross margin).

There is a general differentiation between the partitional and hierarchical classification methods. Simply put, partitional methods try to iteratively minimize the heterogeneity of a given initial allotment of objects into clusters. Hierarchical methods, which are practically significant, take a completely different approach. Initially, each object is located in its own cluster. The objects, however, are then combined successively so that only the smallest level of homogeneity is lost in each step. We can easily present the resulting hierar- chy of nested clusters in a so-called dendrogram.

\subsubsection{Conceptual clustering}

As described above, traditional forms of cluster analysis can identify groups of similar objects but cannot describe these classes beyond a simple list of the individual objects. The objective of many usage scenarios, however, is to characterize the existing structures that are buried among the volumes of data. Instead of representing object classes through simply listing their objects, conceptual clusters intentionally describe them using terms which classify the individual objects through rules. A group of these rules forms a so-called concept.

A basic example of a concept is a program that automatically and logically links individual attribute values. Advanced systems can even establish concepts and concept hierarchies with classification rules.

The different concepts in partitional methods of conceptual clustering compete with each other. Ultimately, we have to choose the clustering concept that best meets the performance criteria for a specific method. Some performance criteria include the simplicity of the concept (based on the number of attributes involved) or the discriminatory power (as the number of variables that have values do not overlap beyond the different object classes.)

Similar to traditional cluster analysis, there are also hierarchical techniques that form classification trees in a topdown approach. As described above, the best classification in terms of performance criteria will take place on each level of the tree. The process ends when no further improvement is possible from one tree 


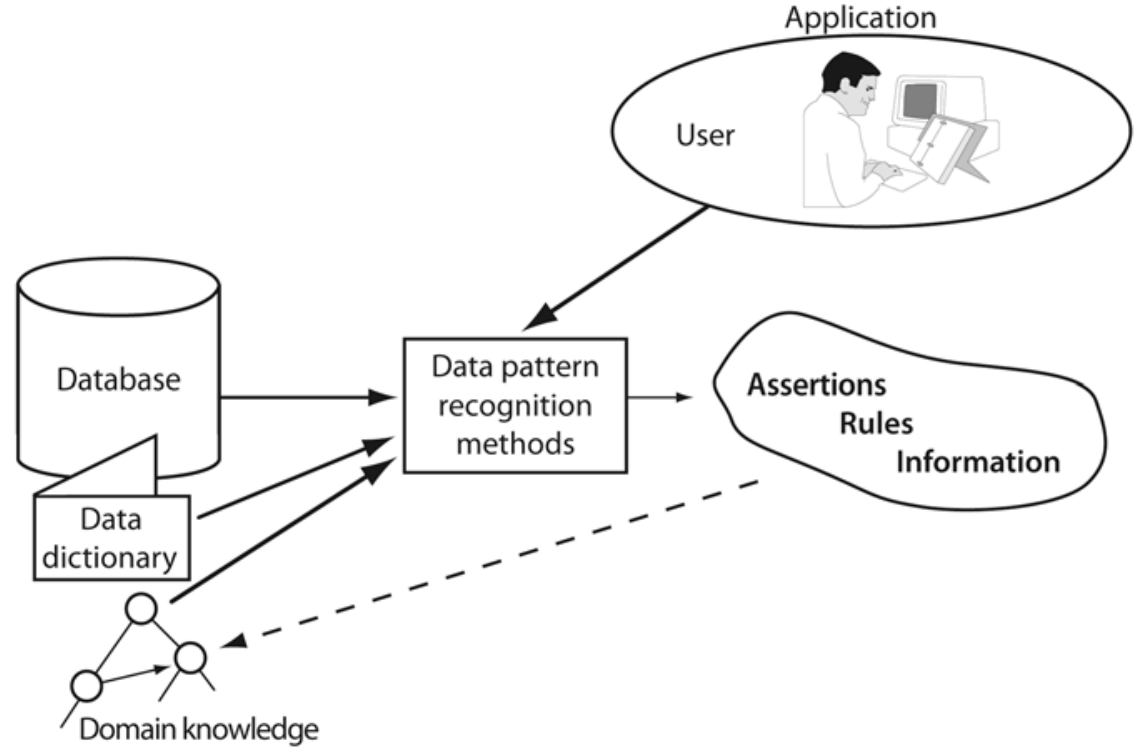

Fig. 3 Framework

level to the next (Fisher and Langley 1986; Stepp and Michalski 1986).

Companies, for example, could use conceptual clustering to classify their customers. Assuming that the customer data records contain information on revenues, gross margin and the number of complaints, a company could create the following model:

- Top customers who together generate more than $50 \%$ of total revenues will be classified as 'A' customers. (A classification can also be made for ' $\mathrm{B}$ ' and 'C' customers.)

- If the marginal profit per unit sale is smaller than a certain threshold, the customer will be classified as one with a 'low gross margin'.

- If the number of complaints exceeds the $20 \%$ average, the customer will be classified as one with a 'high complaint rate'.

- Customers with a 'low gross margin' and 'high complaint rate' are particularly 'bad'.

If a company used the concept above to cluster 'bad' customers, it could help them decide if they should suspend shipments to certain customers due to their constant complaining.

\subsection{Bayesian methods}

Bayesian methods stem from Bayes' famous theorem on conditional probability. Simply stated, these methods attempt to change the classification to maximize the conditional probability that the group matches the actual data structure under the condition of the available data.

The underlying algorithms automatically determine the respective number of object classes and calculate the probability with which the objects belong to classes. Unlike in other procedures, the objects are not strictly classified to a single class. Instead, we can calculate the objects to different groups with various probabilities. In this approach, we assume that the individual clusters distinguish themselves through the probability distributions about the attributes of objects that are given through a model function and its parameter.

Bayesian methods are generally applied in one of two ways:

First, there is a pure classification application which contains all data in its entirety. In this case, we will determine the number of classes, the distribution parameters and the probability that the objects are affiliated to the classes (Cheeseman et al. 1990). However, we can also use these methods when the data is accumulated over a period of time. In this case, we classify the objects in a preliminary class structure in which the distribution parameters were partially determined previously. Here too, we will only know the actual number of classes during the dynamic classification process (Anderson and Matessa 1990).

Let's assume that in the customer classification example above the gross mar- gin per unit sale and the complaint frequency in the potentially available customer groups could shift closer to the normal distribution. Bayesian methods would classify the individual customers with certain probability classes distinguished by means and standard deviations. In particular, companies should thoroughly examine customers that have a high probability of belonging to a class with below-average gross margin or revenues and above-average complaint rates.

\subsection{Inductive learning}

Let's assume that there is a given set of objects (i. e. a training set) with a known class. Inductive learning attempts to define a rule that groups a new object based on its attribute into one of the existing classes. One common approach is to visualize the learned rules as a decision tree ${ }^{3}$. The leaves of the tree represent the classes while the main lower branches represent the tests that respectively check an attribute value. Each possible result of the test receives its own branch that, in turn, either leads into another branch or bears a leaf. The ID3 algorithm, a well-known example of this approach, starts with a section of this training set. In just a few iterations we can build a tree from sets with up to 30,000 objects and up to 50 attributes. ID3 subsequently classifies the remaining objects of the training set. If the classification is incorrect, the algorithm will restart with an extended section of the training set for the objects that have been incorrectly categorized (Quinlan 1986).

Banks, for example, could use a method like this to build and maintain a professional system to check the credit rating of individual customers. If a training set contains a large customer group with either a high or low credit rating, the algorithm can use rules to assess future loan applications which bank employees can process in the system.

\section{Critical factors}

The following section outlines some problems associated with data mining. In our opinion, these critical factors for success will form the foundation for future research and development. 


\subsection{Efficiency of algorithms}

Regarding the efficiency of data mining algorithms, we should consider the following aspects.

- Calculation times are a key factor. If the calculation times of algorithms grow faster than the linear dependency of the squared number of data records to be searched, we could assume that they would not be suitable for larger applications. We can improve calculation times by limiting the search area through user input or reducing the searched data volume through targeted (e. g. user-based) selection and compression. Recent developments show that the calculation time of algorithms will become less relevant due to technical developments (e. g. faster processors, parallel computers).

- The algorithms must be robust enough to deal with incomplete and/or flawed data. The problem here is that flawed data produces noticeable patterns. If a sales region had accidently forgotten to plan revenues for a series of articles, the system should diagnose extremely high budget-actual variances. The system, however, should not present these types of statements as part of the normal analysis results but rather detect them in a plausibility check and report the incomplete sections in a separate report.

\subsection{Expert knowledge}

The rules that are identified through data mining methods must be interpreted. Interpreting this data, however, requires expert knowledge of the area where the data originated. There are many open questions as to which type of knowledge a person needs in order to help interpret the data.

We can debate to which extent the analysis process should be driven - or limited - by expert knowledge. After all, there is a negative connotation between finding "conventional" patterns (i. e. those that were already assumed) and ignoring new, unusual constellations. Some feel it is controversial to use a data dictionary - which describes the attributes, their values, as well as the natural hierarchical relationships between attributes and their values - as applied knowledge. Using this approach, companies could save regions, states, and districts alongside revenue and

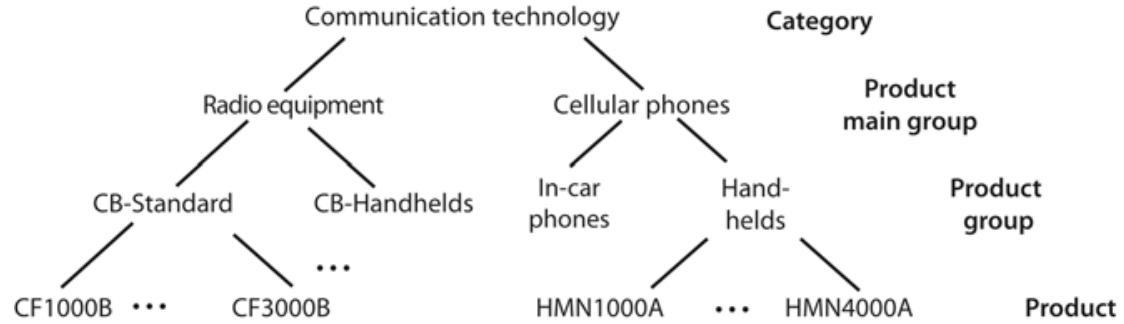

Fig. 4 Data dictionary: relationships between attributes and their values

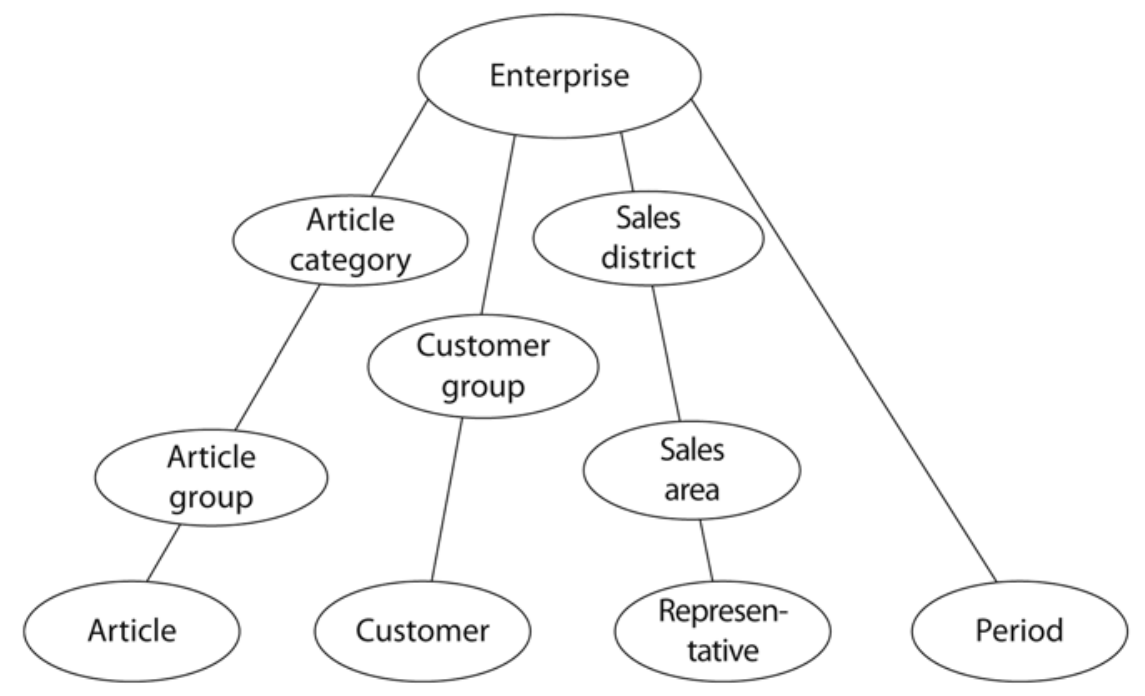

Fig. 5 Partial order

expense data in a profit and loss statement. In this case, the data dictionary would contain information that regions could be divided into states and that Franconia is part of Bavaria. Fig. 4 shows another example of a data dictionary.

\subsection{Dealing with uncertainty}

Most data patterns do not represent certain knowledge. Uncertainty results from faulty or incomplete data or from the fact that the available data do not represent a valid depiction of the real world. As a result, we need to be able to visualize this uncertainty in an appropriate manner. One common approach is to extend the found rules with statistical probability measures such as confidence intervals, standard variance or error measures.

\subsection{Dealing with triviality}

Regarding data mining, triviality occurs in different forms. Some examples include:

- The statement is tautological (e.g. "all pregnant patients were female".)

- Multiple rules describe the same context. (e. g. the statement "The Nurem- berg sales district generated record revenues" would be redundant when the statement "All sales districts in Northern Bavaria generated record revenues" is also true.

- The statement refers to a single element of a random sample.

- Users with expert knowledge have known this statement for some time.

- A simple database query (e. g. sales statistics) could produce the same statement.

Redundancy filters are one way to counter trivial statements. In this case, the elements being examined must have a partial order with clear parent-child-relationships. Redundancy filters in this context are heuristic rules that apply for sections of the partial order. They indicate, for example: "When a statement applies for a parent, eliminate all statements that apply for the children (Hoschka and Klösgen 1991)". As a result, a statement about an article group implies the same statements for a particular article - provided that the individual articles in the group have the same behavior. Fig. 5 illustrates an example of a partial order in gross margin analysis. 


\section{The future course of development}

Hands-on experience shows that the developments in the field of database technologies are posing new challenges for data mining. Typical data mining methods, for example, are not capable of fully utilizing the potential of relational or object-oriented databases. Another very promising usage area appears to be analyzing ever-growing multimedia databases.

Analyzing natural language texts could emerge as another practical usage of data mining in the more distant future. Companies could also deploy applications that support the post-sales follow-up process. If a company processes a large number of complaint reports, it could then use data mining methods to create a special thesaurus. Employees, in turn, could use this thesaurus and standardized templates to formalize these complaints so that others can analyze possible causes of the deficiencies using further data mining methods.

Another interesting area for further research is the development of adaptive systems. These systems automatically adapt their behavior in a self-parameterization that is driven by past operations. We can use these, for example, to limit the detail of future searches if the run time has exceeded a certain threshold. Other ways to regulate the granularity of the analy- sis include the number of delivered statements or the users' acceptance thereof.

Future research must also examine if it is possible to build a filter that is capable of differentiating trivial from non-trivial statements. One conceivable option would be to integrate the user in an incremental learning cycle. In this case, the data mining identification method would still generate statements that are trivial or known to the user (assuming, of course, that the user does not want to limit the analysis too much through individual specifications). To ensure that the system does not continuously present too many trivial statements, administrators could configure the system so that users are prompted to select which rules they found interesting. The remaining trivial statements would then be saved and, ideally, summarized with the rules that were previously marked as not interesting. The system could then process the 'trivial' statements into a special filter so that it would only present 'non-trivial' statements in the future. In certain application areas, this process can be further automated. If the user tends to gather further differentiating information to the interesting statements, for example, the system could automatically monitor and track this information. As a result, the program could automatically determine how interesting the calculated statements are and present them as such.

\section{References}

Anderson JR, Matessa M (1990) A rational analysis of categorization. In: Proceedings of the seventh international conference on machine learning. San Mateo, $\mathrm{p} 76 \mathrm{ff}$

Cheeseman P, Kelly J, Self M, Stutz J, Taylor W, Freeman D (1990) AutoClass: a Bayesian classification system. In: Proceedings of the fifth international conference on machine learning. San Mateo, p $54 \mathrm{ff}$

Fisher D, Langley P (1986) Conceptual clustering and its relation to numerical taxonomy. In: Gale WE (ed) Artificial intelligence and statistics. Reading, $\mathrm{p} 77 \mathrm{ff}$

Frawley WJ, Piatetsky-Shapiro G, Matheus CJ (1991) Knowledge discovery in databases: an overview. In: Piatetsky-Shapiro G, Frawley WJ (eds) Knowledge discovery in databases. Menlo Park

Harmon P (1992) Software trends at the help desk. Intelligent Software 8:1 ff

Hoschka P, Klösgen W (1991) A support system for interpreting statistical data. In: Piatetsky-Shapiro G, Frawley WJ (eds) Knowledge discovery in databases. Menlo Park

Kaufman L, Rousseeuw PJ (1990) Finding groups in data. New York

Quinlan JR (1986) Induction of decision trees. Machine Learning 1(1):81 ff

Quinlan JR (1990) Learning logical definitions from relations. Machine Learning 5(3):239 ff Stepp RE, Michalski RS (1986) Conceptual clustering: inventing goal-oriented classification of structured objects. In: Michalski RS, Carbonell JG, Mitchell TM (eds) Machine learning, vol II. Los Altos, p $471 \mathrm{ff}$ 